\title{
Discontinuous Spectral Difference Method for Conservation Laws on Unstructured Grids
}

\author{
Yen Liu, ${ }^{1}$ Marcel Vinokur, ${ }^{2}$ and Z.J. Wang ${ }^{3}$ \\ 1 NASA Ames Research Center, Moffett Field, CA 94035, USA,Yen. Liu@nasa.gov \\ 2 Eloret Corp., Sunnyvale, CA 94087, USA, vinokur@nas nasa.gov \\ 3 Michigan State University, East Lansing, MI 48824, USA, zjw@egr.msu.edu
}

\section{Summary}

A new, high-order, conservative, and efficient method for conservation laws on unstructured grids is developed. The concept of discontinuous and high-order local representations to achieve conservation and high accuracy is utilized in a manner similar to the Discontinuous Galerkin (DG)[1] and the Spectral Volume (SV)[2] methods, but while these methods are based on the integrated forms of the equations, the new method is based on the differential form to attain a simpler formulation and higher efficiency. Conventional unstructured finite-difference (FD) [3] and finite-volume (FV) [4] methods require data reconstruction based on the least-squares formulation using neighboring point or cell data. Since each unknown employs a different stencil, one must repeat the least-squares inversion for every point or cell at each time step, or store the inversion coefficients. In a high-order, three-dimensional computation, the former would involve impractically large CPU time, while for the latter the memory requirement becomes prohibitive. In addition, the finite-difference method does not satisfy the integral conservation in general. By contrast, the DG and SV methods employ a local, universal reconstruction of a given order of accuracy in each cell in terms of internally defined conservative unknowns. Since the solution is discontinuous across cell boundaries, a Riemann solver is necessary to evaluate boundary flux terms and maintain conservation. In the DG method, a Galerkin finite-element method is employed to update the nodal unknowns within each cell. This requires the inversion of a mass matrix, and the use of quadratures of twice the order of accuracy of the reconstruction to evaluate the surface integrals and additional volume integrals for non-linear flux functions. In the SV method, the integral conservation law is used to update volume averages over subcells defined by a geometrically similar partition of each grid cell. As the order of accuracy increases, the partitioning for 3D requires the introduction of a large number of parameters, whose optimization to achieve convergence becomes increasingly more difficult. Also, the number of interior facets required to subdivide non-planar faces, and the additional 
increase in the number of quadrature points for each facet, increases the computational cost greatly.

In the spectral difference (SD) method, the conservative unknowns in each cell are the number of nodal values required to support a reconstruction of a given order of accuracy. Their locations are chosen so that a quadrature approximation for the volume integral exists at least to the same order of accuracy. The fluxes are calculated at a different set of nodes, whose number will support a reconstruction of one order higher accuracy, since the flux derivatives are used to update the conservative unknowns. They are located so that quadrature approximations for surface integrals over the coll boundaries exist to a required order of accuracy. In addition, the locations of the conservative nodes and the flux nodes must be such that the integral conservation law is satisfied for the cell to the desired order of accuracy. If the nodes are distributed in a geometrically similar manner for all cells, the discretizations become universal, and can be expressed as the same weighted sums of the products of the local metrics and fluxes. These metrics are constants for the line, triangle, and tetrahedron elements, and can be computed analytically for curved elements. We can also show that the number of flux nodes is far less than the number of quadrature points in the SV method. Since all unknowns are decoupled, no mass matrix inversion is required. The SD formulation for the line element is a generalization of the multidomain spectral method[5]. Its tensor products can be used for quadrilateral and hexahedron elements.

\section{The Spectral Difference Method}

The most general form of a conservation law can be written as

$$
\frac{\partial u}{\partial t}+\nabla * F=0
$$

where the conservative variable $u$ can be a scalar or a vector, and the generalized flux $F$ can be a vector or tensor. The term $\nabla * F$ represents the divergence or curi of $F$, depending on the physical definition of $u$. Integrating (1) over cell $i$, we obtain

$$
\frac{\partial}{\partial t} \int_{V_{i}} u d V+\sum_{i=1}^{M} \int_{S_{l, i}} d \mathbf{S} * F=0,
$$

where $V_{i}$ is the volume of the cell $i$, and $S_{l, i}$ is the area of face $l$ for cell $i$. (In $2 D$, each face is actually a line.) Here $M$ is the number of faces, which is one more than the dimension $D$ of the domain.

In each cell, the discrete unknowns are the values of $u$ at quadrature points for the volume integral over the cell. We denote these points, some of which may lie on the cell faces, as $\mathbf{r}_{j, i}$, and define $u_{j, i}$ as $u\left(\mathbf{r}_{j, i}\right)$. The expansion of $u$ in the cell can be written in the cardinal form 


$$
u_{i}(\mathbf{r})=\sum_{j=1}^{N_{n}} L_{j, i}(\mathbf{r}) u_{j, i}
$$

where $L_{j . i}(\mathbf{r})$ are the cardinal basis functions and $N_{n}$ is the number of basis functions required to support a desired degree of precision $n$ of the reconstruction. We will use polynomials as an independent basis. The locations of $\mathbf{r}_{j, i}$ then uniquely define the $L_{j, i}(\mathbf{r})$. In order to evaluate the surface integrals in (2) efficiently, we discretize $F$ at points $\mathbf{r}_{k, i}$, most or all of which are located at quadrature points for those integrals. The expansion of $F$ in the cell can also be written in the cardinal form

$$
F_{i}(\mathbf{r})=\sum_{k=1}^{N_{n+1}} M_{k, i}(\mathbf{r}) F_{k, i}
$$

where $M_{k, i}(\mathbf{r})$ are now the set of cardinal basis functions defined by $\mathrm{r}_{k, i}$ and $F_{k, i}=F\left(\mathbf{r}_{k, i}\right)$. We can satisfy (1) at points $\mathbf{r}_{j, i}$ by evaluating

$$
\nabla * F\left(\mathbf{r}_{j, i}\right)=\sum_{k=1}^{N_{n+1}} \nabla M_{k, i}\left(\mathbf{r}_{j, i}\right) * F_{k, i} .
$$

In order to evaluate $F_{k, i}, u_{k, i}$ is required, which can be obtained directly from (3) as

$$
u_{k, i}=\sum_{j=1}^{N_{n}} L_{j, i}\left(\mathbf{r}_{k, i}\right) u_{j, i} .
$$

To reduce the cost of this interpolation, some of points $\mathbf{r}_{k, i}$ may be chosen to coincide with $\mathbf{r}_{j, i}$. If the points $\mathbf{r}_{j, i}$ and $\mathbf{r}_{k, i}$ are distributed in a geometrically similar manner for all cells and within each cell $i$ the gradient of a function is expressed in terms of its area vectors $S_{i}^{l}$ and volume $V_{i}$, the coefficients in (5) and (6) become universal, independent of cell $i$. There are only a few of these coefficients, which can be calculated exactly and stored in advance. For points $\mathrm{r}_{k, i}$ in the interior of cell; $F_{k, i}=F\left(u_{k, i}\right)$. For those points $\mathrm{r}_{k, i}$ located on the cell faces, since $u$ may be discontinuous, we must replace the flux $F$ by a Riemann flux.

In order to check the integral conservation law for the cell, we must show that

$$
V_{i} \sum_{j=1}^{N_{n}} w_{j} \nabla * F\left(\mathbf{r}_{j, i}\right)=\sum_{l=1}^{M} S_{i}^{l} * \sum_{k} w_{k} F_{k, i} .
$$

Here $w_{j}$ are the volume quadrature weights at the points $\mathbf{r}_{j, i}$ and $w_{k}$ are surface quadrature weights for face $l$, and for each face the summation is over those points $\mathbf{r}_{k, i}$ located on that face. The total contribution from any interior point $F_{k, i}$ to the volume integral in Eq. (7)(righthandside term) must vanish.

From Eqs. (5) and (6), we also see that the SD formulation is very similar to that of the FD method for structured grids. The SD method thus retains the 
simplicity and computational effiency of the structured ED method. However, the metric terms in the latter are eraluated by numerically differencing the grid point coordinates. Since numerical grid generators are mostly only second-order accurate, the overall accuracy of the solution can be severely degraded if the grid is not sufficiently smooth. In contrast, the metric terms in the SD method are computed exactly from the geometry of the grid, no matter how it was generated. It thus retains its formal accuracy, even for very unsmooth unstructured grids. Furthermore, in contrast to the FD method, the integral conservation law is satisfied to the desired accuracy.

\section{Locations of the Unknowns and Flux Points}

The critical part of the SD method is the locations of the $u$ points $\mathbf{r}_{j, i}$ and $F$ points $\mathbf{r}_{k, i}$, which are determined by symmetry groups associated with the cell centroids, vertices, edges, and faces. All but the first contain arbitrary parameters that can be varied to obtain optimum solutions. The number of points required for a reconstruction with a specified degree of precision is greater than the minimum number of Gaussian quadrature points for that precision. One can obtain greater efficiency by locating some $u$ points to coincide with $F$ points: For $F$ points on the vertices in $2 \mathrm{D}$ and edges and vertices in 3D, more than one Riemann solver is necessary. For those formulations with expensive Riemann solvers, these points should be minimized. Another criterion for the placement of $u$ and $F$ points is that the reconstruction matrix is nonsingular. The final criterion is that integral conservation is satisfied within the desired degree of precision. We can show that the number of $F$ points is far less than the number of flux quadrature points in the SV method with the same accuracy.

We first show some representative placements of $u$ points (circles) and $F$ points (squares) that satisfy integral conservation, for various orders of accuracy (which are one more than the degree of precision). Figures $1 \mathrm{a}$ and $1 \mathrm{~b}$ show the placements for line element with second and fifth order of accuracy, and Figures $2 \mathrm{a}, 2 \mathrm{~b}$, and $2 \mathrm{c}$ show the placements for the triangular element with first, second, and third order of accuracy, respectively. Here the $u$ points are placed at the Gaussian quadrature points, while the $F$ points on the surface are placed at Gauss-Lobatto points, except in Fig. 2a, where they are located at the Gaussian points.

\section{Numerical Results}

We first show some one-dimensional convergence studies for the linear convection equation. Plotted are $L_{1}$ (solid lines) an $L_{\infty}$ (dash lines) error norms as functions of orid size for second to ffth order accurate methods. Figure 3a $(1 D-G)$ shows the plots for the $u$ points at the Gaussian quadrature points, 


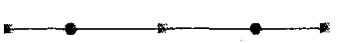

(a) $2^{\text {rdd }}$ order

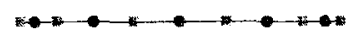

(b) $5^{\text {th }}$ order

Fig. 1. Placement of unknowns and flux points for line element.

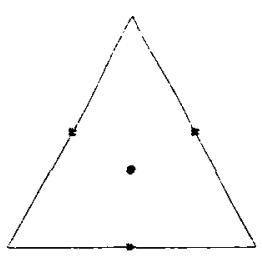

(a) $i^{\text {st }}$ order

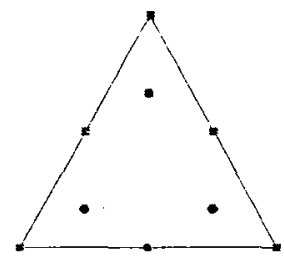

(b) $2^{\text {nd }}$ order

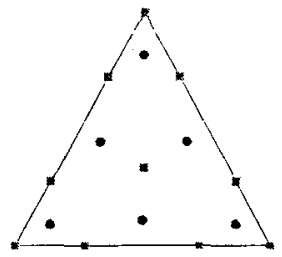

(c) $3^{\text {rd }}$ order

Fig. 2. Placement of unknowns and flux points for triangular element.

while Figure 3b (1D-GL) shows the plots for the $u$ points at the Gauss-Lobatto quadrature points. Both exhibit the expected orders of accuracy. In Fig. 3c (2D) we show analogous plots for a plane wave propagating at 45 degree through a square domain for second and third order accurate methods, using the point placements in Figs. $2 \mathrm{~b}$ and $2 \mathrm{c}$. The third example is the scattering of a TM wave incident on a perfectly conducting circular cylinder. Figure 4 shows the unstructured grid consisting of 2024 triangular cells. The wave propagates from the left to the right with the wave number equal to 5 , based on the radius of the cylinder. This gives approximately 6 cells per wavelength. Contour plots for $E_{z}$ with the exact solution (solid lines) are shown in Figs. $5 \mathrm{a}$, $5 \mathrm{~b}$, and $5 \mathrm{c}$ for first, second, and third order accurate methods, respectively. It is seen that the first order solution is very dissipative with this grid resolution, while the second and third order solutions show an excellent agreement with the exact solution.

\section{References}

1. B. Cockburn and C.W. Shu, J. Comput. Phys., 141, 199-227 (1998).

2. Z.J. Wang and Yen Liu, J. Comput. Phys., 179, 665-697 (2002).

3. D. Sridar and N. Balakrishnan, J. Comput. Phys., 189, 1-29 (2003).

4. T.J. Barth and P.O. Frederichson, AIA.A Paper No. 90-0013, 1990.

5. D.A. Kopriva and J.H Kolias, J. Comput. Phys., 125, 244-261 (1996). 


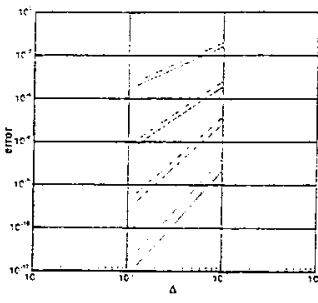

(a) î- $\widetilde{G}$

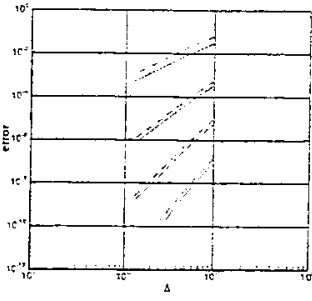

(D) ID-Gi

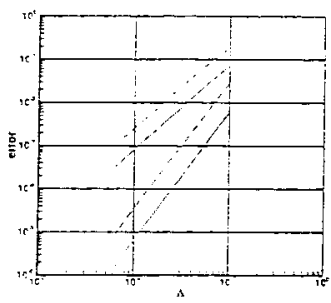

(c) 20

Fig. 3. Error norms of various order of accuracy for line and triangular elements.

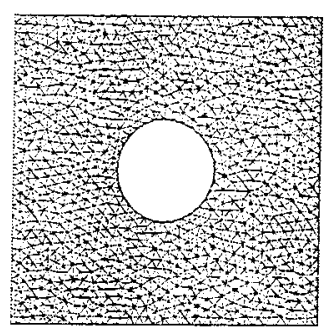

Fig. 4. Grid for a region exterior to a circular cylinder.
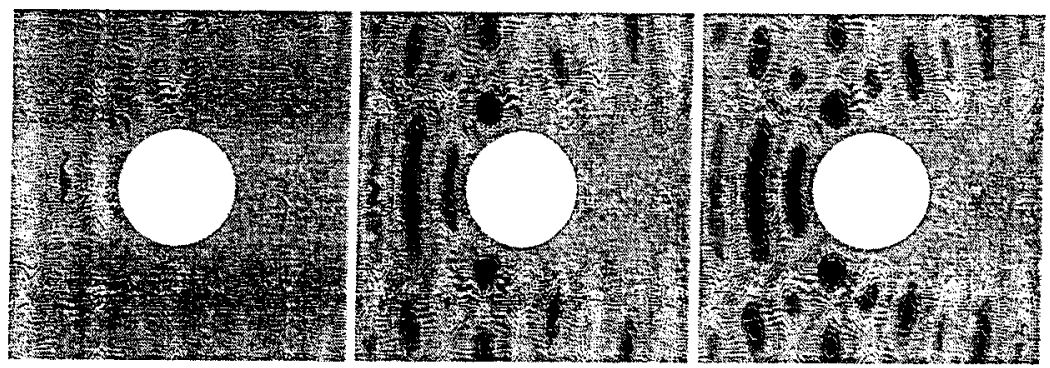
(a) $1^{\text {st }}$ order
(b) $2^{\text {nd }}$ order
(c) $3^{\text {rd }}$ order

Fig. 5. Contour plots of $E_{z}$ for a plane wave incident on a perfectly conducting cylinder. (numerical solutions: color contours, exact solution: solid lines) 